\title{
Probing the origins of vibrational mode specificity in intramolecular dynamics through picosecond time-resolved photoelectron imaging studies
}

Julia A. Davies, Laura E. Whalley and Katharine L. Reid

School of Chemistry, University of Nottingham, Nottingham NG7 2RD, United Kingdom

\begin{abstract}
We have studied the intramolecular dynamics induced by selective photoexcitation of two near-isoenergetic vibrational states in $\mathrm{S}_{1} \mathrm{p}$-fluorotoluene using picosecond time-resolved photoelectron imaging. We find that similar dynamics ensue following the preparation of the $13^{1} 1^{1}$ and $7 a^{1} 1^{1}$ states that lie at $1990 \mathrm{~cm}^{-1}$ and $2026 \mathrm{~cm}^{-1}$, and that these dynamics are mediated by a single strongly coupled doorway state in each case. However, the lifetimes differ by a factor of three, suggesting an influence of the vibrational character of the modes involved. Our results clearly show the contribution of torsion-vibration coupling to the dynamics; this is further corroborated by comparison with the $7 a^{1} 1^{1}$ state in $\mathrm{S}_{1} \mathrm{p}$ difluorobenzene, which lies at $2068 \mathrm{~cm}^{-1}$. We invoke a model in which van der Waals interactions between methyl hydrogen atoms and nearby ring carbon and hydrogen atoms leads to mixing of the vibrational and torsional states. This model predicts that enhanced torsion-vibration coupling occurs when mode $7 \mathrm{a}$ is excited, consistent with our observations.
\end{abstract}

\section{Introduction}

Photochemical processes, so important in Nature and in technology, ${ }^{1}$ are highly dependent on the routes through which energy can be funnelled. Although these routes are dictated by molecular structure, they cannot easily be predicted from theoretical studies. Experimental probes of energy redistribution mechanisms are therefore essential in any quest for the rules of thumb needed to design photochemical systems with desired properties. With this in mind we have pioneered and refined a picosecond time-resolved photoelectron imaging technique that has the capability to provide unique insight into mechanisms of intramolecular energy redistribution. ${ }^{2-6}$

Time-domain experiments typically track the population of an initially prepared "bright state" which can leak out through couplings to one or more dark states. ${ }^{\text {a }}$ The bright state population may oscillate, decay exponentially, or show oscillations superimposed on an exponential decay, depending on the number of dark states involved and the strength of the coupling driving the dynamics. Oscillations in population are a signature of strong coupling between the bright state and specific dark states that mediate the energy redistribution, and the measured oscillation periods are related to energy separations between the eigenstates resulting from the coupling. In cases where the loss of bright state population appears irreversible, radiationless transition theories ${ }^{7}$ invoke Fermi's Golden Rule expression, $\Gamma \alpha \rho<V>^{2}$. This expression correlates the exponential decay rate, $\Gamma$, with the density of

\footnotetext{
${ }^{\text {a }} \mathrm{A}$ bright state is a state that can be accessed by a transition with significant oscillator strength from the ground state. A dark state cannot be accessed by a transition from the ground state.
} 
available dark states, $\rho$, and the square of the average coupling strength, $\langle V\rangle$, to those dark states. However, although this rule holds in some cases ${ }^{8}$ exceptions have been welldocumented for many years, ${ }^{6,9-14}$ and factors such as mode-dependence, ${ }^{15}$ existence of critical "doorway" states, ${ }^{4,6,16}$ molecular flexibility, ${ }^{17,18}$ molecular symmetry ${ }^{5}$ and the nature of substituents ${ }^{8}$ have all been claimed to play a critical role in determining the course of the dynamics.

In order to establish the key factors that influence the rate and mechanism of energy flow it is necessary to conduct comparative studies. ${ }^{6,12,18-21}$ In the present work we consider intramolecular vibrational energy redistribution (IVR) in p-fluorotoluene (pFT) and p-difluorobenzene (pDFB); a comparison made famous by Parmenter and coworkers who used their pioneering chemical timing technique to determine thermally averaged lifetimes of bright states at varying excitation energies. ${ }^{18,22}$ The advantage of studying IVR is that the energy redistributes among levels within the same adiabatic electronic state; it thus provides the simplest prototype for intramolecular dynamics. With our technique we are able to track the population of the bright state, and of specific dark states involved in the redistribution process, by obtaining photoelectron images in 1 ps snapshots after the initial photoexcitation. ${ }^{2-6}$ This in turn enables the determination of mechanistic details concerning the redistribution dynamics. For example, we have previously observed and analyzed quantum beating patterns in photoelectron intensity following the excitation of the $6 a^{1}$ vibrational level that lies at $460 \mathrm{~cm}^{-1}$ internal energy in $\mathrm{S}_{1}$ toluene. ${ }^{2}$ This analysis led to the identification of the relevant dark states, the mechanisms by which they are coupled to the bright state, and the determination of eigenvalue energies and matrix coefficients. Furthermore, we have shown that this technique is highly sensitive to changes in IVR dynamics associated with different torsional levels of the methyl group. ${ }^{2}$

In the present work, we investigate the redistribution dynamics following the preparation of vibrational levels lying at close to $2000 \mathrm{~cm}^{-1}$ in the $S_{1}$ excited state. We prepare the $7 a^{1} 1^{1}$ bright state in $\mathrm{pFT}$, which lies at $2026 \mathrm{~cm}^{-1}$, and compare the results with those from earlier work on the same level in pDFB, ${ }^{5}$ which lies at $2068 \mathrm{~cm}^{-1}$. The $7 \mathrm{a}^{1} 1^{1}$ vibrational level involves excitation of two vibrational modes, 1 and 7a, which are labelled using Varsányi's notation. ${ }^{23, \mathrm{~b}}$ Mode 1 has ring breathing character and mode 7a has $\mathrm{C}-\mathrm{F}$ stretching character; the relevant mode diagrams are shown in Fig. 1. The comparison between the two molecular systems enables us to observe the effect of the attached methyl rotor on the ensuing dynamics. We also prepare the $13^{1} 1^{1}$ bright state in pFT which lies close by in energy $\left(1990 \mathrm{~cm}^{-1}\right.$ in $\left.\mathrm{S}_{1}\right)$ and involves excitation of mode 13 which has $\mathrm{C}-\mathrm{CH}_{3}$ stretching character (see Fig. 1). Study of this level therefore enables us to observe the effect of mode character on the intramolecular dynamics.

\section{Experimental}

\footnotetext{
$\mathrm{b}$ We note that the vibrational modes in pDFB that are denoted 7a and 1 in Varsányi's notation are often referred to as modes 3 and 5, respectively, in Mulliken's notation.
} 
The picosecond laser system (Coherent) and velocity-map imaging photoelectron spectrometer have been described in detail elsewhere. ${ }^{2}$ The two UV outputs from the laser system have pulse durations of $1 \mathrm{ps}$ and bandwidths of $\sim 13 \mathrm{~cm}^{-1}$. The co-propagating laser beams are focused into the photoelectron spectrometer chamber using a $1.0 \mathrm{~m}$ focal length lens, resulting in typical pump and probe pulse intensities of $5 \times 10^{9} \mathrm{~W} / \mathrm{cm}^{2}$ and $1 \times 10^{11} \mathrm{~W} / \mathrm{cm}^{2}$, respectively. The sample of interest, $\mathrm{pFT}$, is seeded in $3 \mathrm{bar} \mathrm{He}$ and expanded through a pulsed nozzle (General Valve) operating at a repetition rate of $40 \mathrm{~Hz}$. The molecular beam is skimmed and a rotational temperature of $\sim 10 \mathrm{~K}$ is achieved. Both laser beams are spatially and temporally overlapped with the pulsed molecular beam inside the spectrometer chamber, which has a typical operating pressure of $4 \times 10^{-8} \mathrm{mbar}$. The pump laser pulse excites the cold molecules to a chosen vibronic state, and a time-delayed probe pulse ionizes the excited molecules. A three-element electrostatic lens focuses the electrons onto a vacuum imaging detector (Photek) to create a two-dimensional image, which is captured using a CCD camera and recorded using IFS32 software (Photek). The pBasex method ${ }^{24}$ is used to reconstruct 3D velocity distributions of the photoelectrons from the measured 2D images and to obtain plots of photoelectron intensity as a function of image radius. Photoelectron spectra can then be realized using the known adiabatic ionization potential of $70946 \mathrm{~cm}^{-1}$ for $\mathrm{pFT} .{ }^{25}$

Three different types of experiment were carried out on pFT, all of which have been discussed in detail elsewhere. ${ }^{2,4}$

(a) Resonant-enhanced multi-photon spectroscopy (REMPI) was employed in order to identify the wavelengths needed to select chosen bright states as well as to establish the shape and width of the absorption profile associated with each bright state. This technique entailed measurement of the total photoelectron signal whilst scanning the pump wavelength from 273.0 to $250.1 \mathrm{~nm}$ over the $S_{0} \rightarrow S_{1}$ absorption profile. The probe wavelength remained fixed at $284.3 \mathrm{~nm}$ and a pump-probe time delay of 10 ps was used.

(b) Slow electron velocity-map imaging (SEVI) was used to obtain plots of photoelectron intensity versus ion internal energy with a mean resolution of $\sim 50 \mathrm{~cm}^{-1}$. This technique provides the best possible energy resolution in the obtained photoelectron spectra but is time-consuming and cannot be used to cover large sets of time delays. In this technique pump wavelengths of 257.40 and $257.15 \mathrm{~nm}$, resonant with the $13^{1} 1^{1}$ and $7 a^{1} 1^{1}$ levels in $S_{1}$, were used along with selected pumpprobe time delays of $0,1,2.5,5,10$ and 100 ps. At each chosen time delay, spectra were measured using six probe wavelengths, which varied from 280.3 to $298.3 \mathrm{~nm}$, and the highest resolution portions of these six spectra were spliced together to produce a single SEVI spectrum.

(c) Two-color photoelectron velocity-map imaging (VMI) was used to obtain photoelectron spectra via the $13^{1} 1^{1}$ and $7 a^{1} 1^{1}$ levels in $S_{1}$ using fixed probe wavelengths of 284.3 and $283.3 \mathrm{~nm}$, respectively. The use of a single probe wavelength results in varying resolution across each spectrum from 130 to $\sim 50 \mathrm{~cm}^{-1}$, but allows much faster data acquisition times than can be obtained using 
SEVI. This enabled spectra to be measured at $\sim 30$ different time delays between 0 and $500 \mathrm{ps.}$

\section{Results}

\section{A. p-fluorotoluene (pFT)}

Figure 2 shows a REMPI spectrum measured across the $S_{0} \rightarrow S_{1}$ absorption profile of pFT. The peaks corresponding to the $13^{1} 1^{1}$ and $7 a^{1} 1^{1}$ vibrational states are identified, as well as those peaks assigned to fundamentals of the contributing modes 1, 13 and 7a. In Figs. 3a and $3 \mathrm{~b}$ we present SEVI spectra measured at selected time delays following excitation of the $\mathrm{S}_{1} 13^{1} 1^{1}$ and $7 \mathrm{a}^{1} 1^{1}$ levels. In each case, the $0 \mathrm{ps}$ SEVI spectrum provides a signature of the initially prepared vibrational wavepacket in $\mathrm{S}_{1}$, which in turn resembles the zero-order bright state $X^{1} 1^{1}$, where $X^{1}=13^{1}$ or $7 a^{1}$. Using the $D_{0}$ vibrational frequencies ${ }^{25}$ and spectroscopic pattern ${ }^{26}$ that have previously been determined for this molecule, it has been possible to assign various peaks in each 0 ps spectrum to vibrational states of the cation. A similar series of peaks is observed in both cases, with the four most intense assigned to $X^{1}, X^{1} 6 a^{1}, X^{1} 1^{1}$ and $X^{1} 1^{2}$. The SEVI spectra observed at later time delays provide signatures of the coupled zeroorder states that are populated as the wavepacket evolves. Each SEVI spectrum therefore provides a snapshot in time of the IVR dynamics that occur in the $S_{1}$ state.

In the SEVI spectra measured at 0 ps the peak assigned to $X^{1} 1^{1}$, which arises from the $\Delta v=0$ transition $S_{1} X^{1} 1^{1} \rightarrow D_{0} X^{1} 1^{1}$, lies at an ion internal energy of $\sim 2055 \mathrm{~cm}^{-1}$ for $13^{1} 1^{1}$ and $\sim 2155 \mathrm{~cm}^{-1}$ for $7 a^{1} 1^{1}$. In both cases, the intensity of the $\Delta v=0$ peak increases from 0 to $2.5 \mathrm{ps}$, but decreases at $5 \mathrm{ps}$. The peak also shifts to $\sim 10 \mathrm{~cm}^{-1}$ higher energy at $2.5 \mathrm{ps}$. From these observations, we deduce that the $\Delta v=0$ transition at $2.5 \mathrm{ps}$ originates not from $\mathrm{S}_{1}$ $X^{1} 1^{1}$, but from ionization of a dark state in $S_{1}$ that is strongly coupled to $X^{1} 1^{1}$. The manifestation of coupling between the bright and dark states is also observed in the relative intensities at 0, 2.5 and 5 ps of the SEVI peak assigned to $X^{1}$ (see Fig. $3 a$ and Fig. 3b). This behavior is characteristic of the bright state population leaking away and then recurring. By contrast, the SEVI spectra at 100 ps are dominated by unstructured signal, comprising unresolved ion states. This is consistent with the ionization of a large number of weaklycoupled dark vibrational states (bath states) that have become populated in $\mathrm{S}_{1}$, and is expected for a statistical IVR process. The observation of time-dependent behavior exhibiting both quantum beating and exponential loss of population has previously been observed by our group ${ }^{4}$ and by others, ${ }^{16}$ and occurs when strongly coupled $\mathrm{S}_{1}$ dark states operate as "doorway states" in the IVR dynamics, mediating the flow of energy away from the bright state and into a bath of dark states. It is the strong coupling between the bright state and the doorway states that gives rise to the quantum beats.

When considering IVR dynamics involving doorway states it is instructive to organize the dark states that could potentially couple to the bright state according to their likely coupling strength, based on the required change in vibrational quanta, the expected energetic proximity and the coupling selection rules. ${ }^{27}$ This arrangement, which gives rise to a tier structure, ${ }^{9}$ enables the identification of likely candidates for the doorway states. The search can be further refined by including the constraint that ionization of a given candidate must 
give rise to a doorway signature peak at the relevant ion energy observed in the SEVI spectra. ${ }^{4}$ We have conducted a search along these lines, using the methodology discussed in detail in Refs. 4 and 6. In the present case we have restricted our search to zero-order states whose energies are predicted to lie within $\pm 25 \mathrm{~cm}^{-1}$ of the required $S_{1}$ energy and $\pm 50 \mathrm{~cm}^{-1}$ of the required $D_{0}$ energy. Surprisingly, given the relatively high density of states at this level of excitation, only two states appear in the lowest tier, one of which shows much closer agreement with the required $D_{0}$ energy. This is the $X^{1} 9 b^{2}$ vibrational state, whose population requires a change of only three vibrational quanta and which is predicted to lie $\sim 4 \mathrm{~cm}^{-1}$ from the $X^{1} 1^{1}$ bright state in $S_{1}$. Further support for this assignment is provided by the observation of strong anharmonic coupling between $1^{1}$ and $9 b^{2}$ in a previous study. ${ }^{3}$ Accordingly, we assign the doorway state to $X^{1} 9 b^{2}$ and have added corresponding ion state assignments to the 2.5 ps spectra in Figure 3 . The vibrational motion associated with mode $9 \mathrm{~b}$ is shown in Fig. 1.

\section{B. Comparison with p-difluorobenzene (pDFB)}

Figures 4 and 5 show two-color photoelectron spectra that were measured for pFT following excitation of the $13^{1} 1^{1}$ (Figs. 4a, 5a) and $7 a^{1} 1^{1}$ (Figs. 4b, 5b) vibrational states in $S_{1}$ at a selection of time delays, together with equivalent photoelectron spectra measured previously for pDFB via the $S_{1} 7 a^{1} 1^{1}$ state (Figs. 4c, 5c). In each of the three cases the five most intense peaks have been labeled from $A$ to $E$ in the 0 ps spectra. The energy of each peak is given in Table I along with the assignment of the ion state that dominates each peak. It is interesting to note that a small amount of unstructured signal is observed below the peaks in the 0 ps spectra for $\mathrm{pFT}$, which is not present for pDFB; this signal is particularly pronounced for the $S_{1} 7 a^{1} 1^{1}$ level in pFT (Figs. $4 b, 5 b$ ). The presence of unstructured signal at 0 ps suggests that some of the eigenstates associated with weakly coupled bath states lie outside of the laser spectral profile (see Section 4A).

In all three cases (see Fig. 4), oscillations in the peak intensities are seen to occur during the first 6 ps. In the two pFT cases (Figs. 4a, 4b), the intensities of peaks A, B and E decrease between 0 and 2 ps whilst peaks $C$ and $D$ increase. At 5 ps the spectral profiles resemble those seen at $0 \mathrm{ps}$, albeit with lower peak intensities and increased congestion; this is consistent with an oscillation period of $\sim 5 \mathrm{ps}$, as discussed in the previous section. The pFT photoelectron spectra measured at longer times (Figs. 5a and 5b) have lost most of their resolved structure by $30 \mathrm{ps}$. Spectral congestion at long time delays was also observed in the higher resolution SEVI spectra shown in Fig. 3. The fact that the congestion is more pronounced at $30 \mathrm{ps}$ for the $7 \mathrm{a}^{1} 1^{1}$ level is consistent with a shorter IVR lifetime for this level compared with $13^{1} 1^{1}$; this is discussed further in Section 4B. In the case of pDFB (Fig. 4c) the intensities of peaks $A$ and $E$ decrease between 0 and 4 ps, whilst that of peak $B$ increases; the intensities of the other peaks show more subtle changes. This behavior has been discussed in Ref. ${ }^{5}$ and is found to be the early time signature of a wavepacket undergoing extended quantum beating that includes an oscillation period of $\sim 8 \mathrm{ps}$. Importantly, the photoelectron spectra have well-resolved structure for many hundreds of picoseconds (see Fig. 5c), and therefore the IVR mechanism involves energy redistribution among a restricted set of coupled vibrational states. ${ }^{5}$ 


\section{IVR timescales}

(i) p-fluorotoluene

In order to gain accurate values for the oscillation periods and exponential decay times for the two pFT levels, the areas of peaks A to $E$ that lie above the unstructured signal in the photoelectron spectra have been determined using the standard trapezoidal rule, at all measured time delays. The areas of peaks $A$ and $C$ predominantly reflect the populations of the bright and doorway states in $S_{1}$, and their time profiles are shown by the data points in the top and middle panels of Figs. $6 \mathrm{a}$ and $6 \mathrm{~b}$. The areas of three unstructured spectral regions that lie in between the peaks have also been deduced; these areas represent the time-dependent growth of the bath state population and examples are shown in the bottom panels of Figs. $6 a$ and $6 \mathrm{~b}$.

The time-dependent intensity, $I(t)$, for each peak A-E is fitted to the empirical equation;

$$
I(t)=a+b \exp \left(\frac{-t}{\tau_{b}}\right)+c \exp \left(\frac{-t}{\tau_{c}}\right) \cos \left(\frac{2 \pi t}{\tau_{o s c}}\right)
$$

Examples of the fit results are shown by the solid lines in the upper and middle panels in Figs. $6 a$ and $6 \mathrm{~b}$. The values determined for $\tau_{b}$ and $\tau_{c}$ give a measure of the irreversible IVR lifetime, $\tau_{\mathrm{IVR}}$, whilst $\tau_{\mathrm{osc}}$ reflects the oscillation periods associated with the reversible transfer of vibrational state population between the bright and doorway states in $\mathrm{S}_{1}$. The values determined for $a, b$ and $c$ reflect the total photoelectron intensity and will be different for each peak, but because the values of $\tau_{b}, \tau_{c}$, and $\tau_{\text {osc }}$ reflect the IVR dynamics they should be the same for each set of peaks A-E. Therefore, the time constants can be accurately determined even though the photoelectron peaks encompass unresolved ion vibrational states. The empirical equation used to provide fits to the unstructured spectral regions is similar to Eq. 1, but it omits the last term and therefore provides values for $\tau_{b}$ but not for $\tau_{c}$ or $\tau_{\text {osc. }}$ Examples of these fit results are shown by the solid lines in the lower panels of Fig. $6 a$ and $6 b$.

For each of the two levels studied in $\mathrm{S}_{1} \mathrm{pFT}$, we take $\tau_{\mathrm{IVR}}$ to be the average of the ten values of $\tau_{b}$ and $\tau_{c}$ determined for the photoelectron peaks A-E plus the three values of $\tau_{b}$ determined for the three chosen unstructured spectral regions. The standard deviation of these 13 values is taken to be the error bar on $\tau_{\text {IVR. }}$. An average value of $\tau_{\text {osc }}$ is determined for the five photoelectron peaks $A-E$ and is related to the energy separation, $\Delta E_{12}$, between the two eigenstates containing the largest contributions from the bright state and the doorway state, according to the equation $E_{12}=h /_{\text {osc }}^{-}$. In Table II, the deduced values for $\tau_{\mathrm{IVR}},{ }_{\text {osc }}$ and $\Delta E_{12}$ are presented for the two $\mathrm{S}_{1} \mathrm{pFT}$ levels $13^{1} 1^{1}$ and $7 \mathrm{a}^{1} 1^{1}$. Comparison with the $\tau_{\mathrm{IVR}}$ value determined for the $7 \mathrm{a}^{1} 1^{1}$ level by Parmenter and coworkers ${ }^{12,22}$ for a room temperature sample has also been included.

\section{(ii) p-difluorobenzene}


In the case of pDFB, the time behavior of the IVR dynamics for the $S_{1} 7 a^{1} 1^{1}$ bright state has already been analyzed in Ref. 5; the previously deduced ${ }_{\text {osc }}^{-}$values are given in Table II. The value of $\tau_{\text {IVR }}$ is taken to be infinity because this level does not appear to undergo statistical IVR to a bath of dark vibrational states, although on a much longer timescale it will lose population through fluorescence. We also note that rotational dephasing ${ }^{28}$ causes the oscillation amplitudes to decrease on a timescale of $\sim 400 \mathrm{ps}$. The $\tau_{\text {IVR }}$ value of $97 \mathrm{ps}$ determined by Parmenter and coworkers for a room temperature sample has also been included for comparison. ${ }^{12,22}$

In Fig. $6 \mathrm{c}$ we show time profiles of three photoelectron peak areas for pDFB, for comparison with the pFT data shown in Figs. $6 \mathrm{a}$ and $6 \mathrm{~b}$. The top and middle panels of this figure show the time profiles for peaks $A$ and $B$. Even though the oscillation amplitude decreases between 0 and 40 ps we know from our previous work that it increases again at later time delays, ${ }^{5}$ as a consequence of the dynamics of a complex beating pattern which persists for $>1 \mathrm{~ns}$. This is in contrast to the oscillation amplitude seen for the pFT levels, which continues to decrease as the time delay gets longer. In the lowest panel of Fig. $6 \mathrm{c}$, we present the time profile for a pDFB photoelectron peak at $2550 \mathrm{~cm}^{-1}$, illustrating the presence of an oscillatory component with a relatively long period of $103 \mathrm{ps}$. The shape of this profile is distinctly different from the exponential increase in signal observed for pFT in the lower panels of Figs. $6 \mathrm{a}$ and $6 \mathrm{~b}$. The solid lines in Fig. $6 \mathrm{c}$ represent fits of the data to a series of cosine terms with oscillation periods corresponding to the $\bar{\tau}_{\text {osc }}$ values given in Table II.

\section{Discussion}

\section{A. Mode-dependence: $13^{1} 1^{1}$ and $7 a^{1} 1^{1}$ in $S_{1} p$-fluorotoluene}

Comparison of the results for the $13^{1} 1^{1}$ and $7 a^{1} 1^{1}$ levels in $S_{1}$ pFT reveals a number of similarities. Firstly, the IVR process is mediated by a doorway state in both cases, as discussed in Section 3A. Secondly, assignment of the ion states observed in the SEVI spectra leads to the conclusion that the doorway state can be assigned to $X^{1} 9 b^{2}$ in both cases. Thirdly, the oscillation period observed for both levels is $\sim 5 \mathrm{ps,}$, corresponding to an eigenstate separation of $\sim 6.7 \mathrm{~cm}^{-1}$. However, a notable difference is that the IVR lifetimes differ by a factor of approximately three; from $\sim 18$ ps for $S_{1} 13^{1} 1^{1}$ to $\sim 7$ ps for $S_{1} 7 a^{1} 1^{1}$. Because the two $S_{1}$ levels have very similar internal energies $\left(\sim 30 \mathrm{~cm}^{-1}\right.$ apart at an internal energy of $\sim 2000 \mathrm{~cm}^{-1}$ ), and therefore lie in a region of similar state density, any differences in the IVR mechanism or lifetime is most likely caused by a dependence on the vibrational character of mode $X$. Thus, we infer that mode 7a promotes IVR more effectively than mode 13.

As mentioned in Section 3B, the unstructured photoelectron intensity that appears at $0 \mathrm{ps}$ is more pronounced for $7 a^{1} 1^{1}$ than for $13^{1} 1^{1}$. This could be explained if a larger proportion of the contributing eigenstates for $7 a^{1} 1^{1}$ lie outside the laser spectral profile. Such an explanation is consistent with the shorter lifetime determined for $7 a^{1} 1^{1}$ in the present work and with the widths of peaks observed in an absorption spectrum measured by Parmenter's group. ${ }^{29}$ Because the eigenstates responsible for the unstructured signal at 0 ps must be 
widely separated in energy we consider what this tells us about the coupling interaction giving rise to them. For simplicity, we consider the coupling of a pair of zero-order states with energies $E_{a}$ and $E_{b}$. The resulting eigenstate energy separation, $\Delta E_{12}$, is given by:

$$
E_{12}=\left(E_{a b}^{2}+4 V_{a b}^{2}\right)^{1 / 2}
$$

where $\Delta E_{a b}=E_{a}-E_{b}$ and $V_{a b}$ is the Hamiltonian coupling matrix element. Therefore, eigenstates that are widely separated in energy are associated with large coupling matrix elements $\left(V_{a b}\right)$ and/or large zero-order energy separations $\left(\Delta E_{a b}\right)$. Furthermore, when $\Delta E_{12}$ is significantly greater than the laser bandwidth $\left(\Delta E_{12}>13 \mathrm{~cm}^{-1}\right.$ in this case) such that only one of the eigenstates is excited, then the initially excited state will contain contributions from both the bright and dark zero-order states and there can be no time dependence associated with the coupling of the two zero-order states. These ideas are discussed in detail by Felker and Zewail ${ }^{30}$ and are illustrated in Fig. 7. The principles still remain when multiple zeroorder levels are involved, as in the specific cases considered here, and will be utilized in the interpretation of our results.

In Figs. $6 \mathrm{a}$ and $6 \mathrm{~b}$ the time profiles show only one resolved oscillation period. This period is associated with the strong coupling between the bright state, $\mathrm{X}^{1} 1^{1}$, and the doorway state, $X^{1} 9 b^{2}$; coupling to any other zero order states must therefore be weaker than this. We can obtain a reasonable estimate of $V_{a b} \sim 3.0 \mathrm{~cm}^{-1}$ for the strong coupling limit by using results from our previous work on the $1^{1} / 9 \mathrm{~b}^{2}$ Fermi resonance in $\mathrm{S}_{1} \mathrm{pFT} .^{2}$ Using this limit, together with the relationship shown in Eq. 2, we show in Fig. 8 that we require $\Delta E_{a b}>11 \mathrm{~cm}^{-1}$ in order to achieve $\Delta \mathrm{E}_{12}>13 \mathrm{~cm}^{-1}$. We can thus deduce that large energy separations between zero-order coupled states are responsible for the exponential decays in bright state population observed for both $\mathrm{pFT}$ levels. This in turn means that the faster IVR dynamics observed for $7 a^{1} 1^{1}$ compared with $13^{1} 1^{1}$ result from larger values of $\Delta E_{a b}$, i.e., from coupling to zero-order bath states that are farther away in energy from the bright state.

In order to explore further the mode-dependence of the IVR dynamics, we invoke additional experimental work in which we have prepared $S_{1} \mathrm{pFT}$ in other vibrational levels that involve the contributing modes 1,13 and $7 a^{3,6}$ In Fig. 9 time profiles of the $\Delta v=0$ photoelectron peaks are shown following excitation of the $1^{1}, 13^{1}$ and $7 a^{1}$ bright states; these states lie at $\mathrm{S}_{1}$ internal energies of $\sim 800,1194$ and $1230 \mathrm{~cm}^{-1}$, respectively. The observed time profiles contain some features in common with those observed for the $X^{1} 1^{1}$ levels (Figs. 6a and 6b). For example, the 5 ps oscillations observed for both $X^{1} 1^{1}$ bright states are also present for the $1^{1}$ bright state (Fig. 9a). Similarly, the overall exponential decay of population observed for both $X^{1} 1^{1}$ bright states is also a feature of the time profiles observed for each $X^{1}$ bright state (Figs. 9b and 9c). Furthermore, the exponential decay timescale for level $X^{1}$ is shorter by a factor of approximately four when $X=7$ a compared with when $X=13$ (see Table III). This provides further evidence that mode 7 a enhances IVR in $\mathrm{pFT}$.

The key timescales extracted from all the pFT time profiles shown in Figs. 6 and 9 are summarized in Table III. It is particularly interesting to compare the $\tau_{\text {IVR }}$ values determined 
for the $7 a^{1}$ and $13^{1} 1^{1}$ levels in $S_{1}$ pFT. The $7 a^{1}$ level lies at an internal energy of $1230 \mathrm{~cm}^{-1}$, in a region of density of states $\sim 10$ times lower than the corresponding region for the $13^{1} 1^{1}$ level which lies at $1990 \mathrm{~cm}^{-1}$. According to Fermi's Golden Rule, the IVR lifetime of $\mathrm{S}_{1} 7 \mathrm{a}^{1}$ should be $\sim 10$ times longer than the IVR lifetime of $S_{1} 13^{1} 1^{1}$, assuming similar average coupling strengths in both cases. However, the measured IVR lifetime for the $7 a^{1}$ level (13 ps) is actually shorter than that of the $13^{1} 1^{1}$ level (18 ps); see Table III. This observation is again consistent with mode 7a being significantly more effective than mode 13 at promoting IVR in pFT. We discuss the reasons for this in Section 4C.

\section{B. Influence of methyl rotor: $7 a^{1} 1^{1}$ in $S_{1} p$-fluorotoluene and $S_{1} p$-difluorobenzene}

In order to understand the role of the methyl group in accelerating IVR, we compare the $7 a^{1} 1^{1}$ photoelectron spectra from pFT and pDFB (see Figs. $4 b$ and $4 c$ ). In the case of pDFB, we have previously shown that restricted IVR dynamics occur that are controlled by strong anharmonic coupling between the bright state and three dark states. These dark states have been assigned to $7 a^{1} 6 a^{2}\left(3^{1} 6^{2}\right), 1^{1} 11^{1}\left(5^{1} 28^{2}\right)$ and $6 a^{2} 11^{2}\left(6^{2} 28^{2}\right)$, where the Mulliken labels commonly used for pDFB are given in parentheses. ${ }^{5}$ Extended quantum beating of the bright state population occurs for at least $1 \mathrm{~ns}$ with no overall loss to bath states; an unpassable bottleneck is reached. By contrast, we have seen in the present work that in the case of pFT population leaks away from the bright state with a deduced lifetime of only $7 \mathrm{ps.}$ The IVR dynamics that result from excitation of $7 a^{1} 1^{1}$ are therefore dramatically different in the two molecules.

The advantage of the sensitivity of our technique to quantum beating effects can be seen in Figs. $6 \mathrm{~b}$ and $6 \mathrm{c}$ in which beating appears as a prominent feature of the time profiles shown. Because the bright state is strongly coupled through anharmonicity to a doorway state in both molecules, it must be the coupling to the bath of available dark states that differs so dramatically in the two cases. On first inspection, it seems likely that the difference arises primarily from the restriction in the number of states that are available for coupling in pDFB as a consequence of the higher molecular symmetry and lack of torsional mode. However, in pDFB the density of available states with the correct symmetry is $\sim 8$ per $\mathrm{cm}^{-1}$, and states separated by many $\mathrm{cm}^{-1}$ can in principle be coupled through anharmonicity. ${ }^{31}$ It would be reasonable to expect, therefore, that $>100$ states are available to couple to the bright state. The fact that only three of these make a significant contribution suggests that weak anharmonic coupling does not occur in pDFB, which in turn suggests that it is unlikely to occur in pFT.

As we remarked in Section $3 \mathrm{~B}$, any unstructured signal in the photoelectron spectrum at $0 \mathrm{ps}$ is negligible for the $7 a^{1} 1^{1}$ level in pDFB by comparison with that observed for either of the pFT levels; therefore we deduce that anharmonic coupling alone does not lead to spectral congestion. This in turn suggests that the influence of torsion-vibration coupling must account for the spectral congestion observed in the pFT photoelectron spectrum at $0 \mathrm{ps}$. If so, we can deduce that mode 7 a facilitates torsion-vibration coupling to zero-order states that are far from the bright state. We therefore conclude that strong anharmonic coupling to just a few dark states is a feature for both pDFB and pFT, weak anharmonic coupling is not 
a feature for either, but weak torsion-vibration coupling to a large number of dark states occurs for $\mathrm{pFT}$. The absence of a methyl rotor therefore severely restricts the IVR dynamics.

\section{Role of torsion-vibration coupling in mode-dependence}

We have demonstrated that torsion-vibration coupling is the mechanism responsible for the irreversible IVR observed for the $7 \mathrm{a}^{1} 1^{1}$ level in $\mathrm{pFT}$ and we have deduced that this mechanism enables coupling to dark states lying outside the laser spectral profile. We can therefore infer that torsion-vibration coupling is also the mechanism responsible for coupling to a bath of states for the $13^{1} 1^{1}$ level in pFT. Further evidence for the role played by the methyl rotor for levels containing mode 13 can be obtained from the time profile shown in Fig. $8 \mathrm{~b}$ following excitation of the $13^{1}$ level at $\sim 1194 \mathrm{~cm}^{-1}$. This plot shows that the bright state population only reduces by $50 \%$ over the whole IVR timescale. This is consistent with only one torsional component undergoing IVR, which in turn is consistent with the IVR being driven by a torsion-vibration coupling mechanism. This phenomenon has been discussed in more detail in a previous publication. ${ }^{6}$

In order to understand why vibrational states involving mode 7a should have a higher propensity for torsion-vibration coupling than vibrational states involving mode 13 we refer back to the atomic displacements illustrated in Fig. 1. In order for torsion-vibration coupling to occur there needs to be an interaction between the methyl group and other atoms in the molecule. It is pertinent to ask therefore what mechanisms are responsible for this interaction. Experimental work from Borst and Pratt ${ }^{32}$ and computational work from Nakai and Kawai ${ }^{33}$ supports the idea that a hyperconjugative effect is responsible, but such an effect does not provide a clear reason for the mode-dependence that we have observed. In earlier work Moss et al. developed a simple model of the interaction between the methyl group and the aromatic ring and used it to correctly predict the size of the torsional barrier and, moreover, to account for spectral congestion observed at low energies in $\mathrm{S}_{1} \mathrm{pFT} .{ }^{34} \mathrm{In}$ this model the interaction results from net van der Waals repulsion between the methyl hydrogen atoms and nearby carbon and hydrogen atoms attached to the aromatic ring. Accordingly, the vibrational motion leads to modulation of the height and shape of the sixfold torsional potential, enabling mixing of the ring vibrational states and the methyl rotor states. Here we extend this model to consider the relative interaction energies that would be expected when different vibrational modes are excited.

In order to characterize the geometry and vibrational modes of pFT in its $\mathrm{S}_{1}$ electronic state, we have conducted calculations at the CIS/6-311G** level of theory, consistent with those discussed in our earlier work, ${ }^{3}$ with the methyl group in the lowest energy configuration, i.e., staggered with respect to the plane of the aromatic ring. These calculations have enabled us to determine the $\mathrm{H}_{m}-\mathrm{H}_{r}$ and $\mathrm{H}_{m}-\mathrm{C}_{r}$ separations between each methyl hydrogen atom $\left(\mathrm{H}_{m}\right)$ and nearby atoms on the aromatic ring $\left(\mathrm{H}_{\mathrm{r}}\right.$ and $\left.\mathrm{C}_{\mathrm{r}}\right)$ at the two extremes of vibrational motion associated with each of the modes 1,13 and $7 \mathrm{a}$. This in turn has enabled us to calculate the net van der Waals interaction energy, $E_{\mathrm{vdw}}$, between the methyl rotor and the ring, which is dominated by interactions between those $\mathrm{H}_{m}$ and $\mathrm{H}_{r} / \mathrm{C}_{r}$ atoms that are closest to each other at each extreme of motion. The pertinent results of these calculations, which use the van der Waals parameters and equations provided by Moss et al, ${ }^{34}$ are summarised in Table IV. 
The maximum $E_{\mathrm{vdw}}$ value calculated for mode $1\left(215 \mathrm{~cm}^{-1}\right)$ is little different from the value calculated at equilibrium $\left(137 \mathrm{~cm}^{-1}\right)$. By contrast, the maximum $E_{\mathrm{vdw}}$ value for mode $7 \mathrm{a}$ $\left(1417 \mathrm{~cm}^{-1}\right)$ is more than 10 times higher than the equilibrium value and $19 \%$ higher than the equivalent value of $E_{\mathrm{vdw}}$ for mode $13\left(1187 \mathrm{~cm}^{-1}\right)$. Even more significantly, as the nuclei move when mode $7 \mathrm{a}$ is excited the van der Waals interaction energy changes by $1463 \mathrm{~cm}^{-1}$, which is $32 \%$ higher than the value of $1111 \mathrm{~cm}^{-1}$ for mode 13 . Thus, a particularly significant modulation of the torsional potential occurs during the vibrational motion associated with mode $7 a$, leading to enhanced mixing of the ring vibrational states and the methyl rotor states.

A simple van der Waals model therefore predicts that vibrational states involving mode $7 \mathrm{a}$ are particularly susceptible to torsion-vibration coupling. Such a prediction is consistent with our observation that the IVR lifetime associated with $7 a^{1} 1^{1}$ is shorter by a factor of $\sim 3$ than the IVR lifetime of the nearly isoenergetic level $13^{1} 1^{1}$, with which the $7 a^{1} 1^{1}$ level shares many characteristics. It is also consistent with the relative IVR lifetimes associated with $7 a^{1}$ and $13^{1}$. (See Table III).

\section{Conclusion}

Similar dynamics in $\mathrm{S}_{1} \mathrm{pFT}$ ensue following the photoexcitation of the $13^{1} 1^{1}$ state at $1990 \mathrm{~cm}^{-1}$ and the $7 \mathrm{a}^{1} 1^{1}$ state at $2026 \mathrm{~cm}^{-1}$, with the IVR dynamics mediated by a single strongly coupled doorway state in each case. The doorway states have been assigned to $13^{1} 9 b^{2}$ and $7 a^{1} 9 b^{2}$, and IVR lifetimes of 18 and $7 \mathrm{ps,} \mathrm{respectively,} \mathrm{have} \mathrm{been} \mathrm{determined.}$ This difference in lifetime cannot be explained by differences in the total densities of states, and evidence of mode-dependent dynamics has been provided, where the promoting role played by mode $7 a$ is related to its enhanced capability to couple via a torsion-vibration mechanism to zero-order dark states that have energies far from the bright state. This behavior is consistent with the promotion of torsion-vibration mixing by a van der Waals interaction between the methyl group and the aromatic ring. In contrast to the short IVR lifetime measured via the $7 a^{1} 1^{1}$ level in $\mathrm{pFT}$, restricted IVR is observed following the preparation of the same level in pDFB. This difference is consistent with the loss of the torsion-vibration coupling route to energy redistribution in pDFB.

\section{Acknowledgements}

We would like to thank Jonathan Midgley for his previous work on the pDFB data. We are also grateful to Anna Andrejeva and Carolyn Withers for their involvement in the preliminary analysis of some of the pFT data presented here. This work was supported by EPSRC grant EP/E046150.

\section{References}

1. G. R. Fleming and M. A. Ratner, Phys. Today 61, 28 (2008).

2. J. A. Davies, A. M. Green and K. L. Reid, Phys. Chem. Chem. Phys. 12, 9872 (2010).

3. J. A. Davies and K. L. Reid, Phys. Rev. Lett. 109, 193004 (2012).

4. J. A. Davies and K. L. Reid, J. Chem. Phys. 135, 124305 (2011).

5. J. Midgley, J. A. Davies and K. L. Reid, J. Phys. Chem. Lett. 5, 2484 (2014). 
6. J. A. Davies, A. M. Green, A. M. Gardner, C. D. Withers, T. G. Wright and K. L. Reid, Phys. Chem. Chem. Phys. 16, 430 (2014).

7. P. Avouris, W. M. Gelbart and M. A. El-Sayed, Chem. Rev. 77, 793 (1977).

8. R. S. von Benten, Y. X. Liu and B. Abel, J. Phys. Chem. A 114, 11522 (2010).

9. K. K. Lehmann, G. Scoles and B. H. Pate, Annual Rev. Phys. Chem. 45, 241 (1994).

10. J. W. Dolce, A. Callegari, B. Meyer, K. K. Lehmann and G. Scoles, J. Chem. Phys. 107, 6549 (1997).

11. Y. Yamada, T. Ebata, M. Kayano and N. Mikami, J. Chem. Phys. 120, 7400 (2004).

12. D. B. Moss and C. S. Parmenter, J. Chem. Phys. 98, 6897 (1993).

13. D. S. Perry, J. Martens, B. Amyay and M. Herman, Molecular Phys. 110, 2687 (2012).

14. A. L. Malinovsky, A. A. Makarov and E. A. Ryabov, Physica Scripta 85, 058102 (2012).

15. S. H. Kable, W. D. Lawrance and A. E. W. Knight, J. Phys. Chem. 86, 1244 (1982).

16. Y. Yamada, N. Mikami and T. Ebata, J. Chem. Phys. 121, 11530 (2004).

17. G. A. Bethardy, X. L. Wang and D. S. Perry, Canadian J. Chem. 72, 652 (1994).

18. C. S. Parmenter and B. M. Stone, J. Chem. Phys. 84, 4710 (1986).

19. J. E. Gambogi, J. H. Timmermans, K. K. Lehmann and G. Scoles, J. Chem. Phys. 99, 9314 (1993).

20. E. R. T. Kerstel, K. K. Lehmann, T. F. Mentel, B. H. Pate and G. Scoles, J. Phys. Chem. 95, 8282 (1991).

21. P. J. Timbers, C. S. Parmenter and D. B. Moss, J. Chem. Phys. 100, 1028 (1994).

22. K. W. Holtzclaw and C. S. Parmenter, J. Chem. Phys. 84, 1099 (1986).

23. G. Varsanyi, Assignments for Vibrational Spectra of Seven Hundred Benzene Derivatives. (Wiley, New York, 1974).

24. G. A. Garcia, L. Nahon and I. Powis, Rev. Scientific Instruments 75, 4989 (2004).

25. V. L. Ayles, C. J. Hammond, D. E. Bergeron and T. G. Wright, J. Chem. Phys. 126, 244304 (2006).

26. S. M. Bellm, P. T. Whiteside and K. L. Reid, J. Phys. Chem. A 107, 7373 (2003).

27. D. J. Nesbitt and R. W. Field, J. Phys. Chem. 100, 12735 (1996).

28. P. M. Felker and A. H. Zewail, J. Chem. Phys. 82, 2994 (1985).

29. Q. Ju, C. S. Parmenter, T. A. Stone and Z. Q. Zhao, Isr. J. Chem. 37, 379 (1997).

30. P. M. Felker and A. H. Zewail, J. Chem. Phys. 82, 2961 (1985).

31. N. T. Whetton and W. D. Lawrance, J. Phys. Chem. 93, 5377 (1989).

32. D. R. Borst and D. W. Pratt, J. Chem. Phys. 113 (9), 3658 (2000).

33. H. Nakai and M. Kawai, J. Chem. Phys. 113, 2168 (2000).

34. D. B. Moss, C. S. Parmenter and G. E. Ewing, J. Chem. Phys. 86, 51-61 (1987). 
Table I: Ion internal energies $\left(\mathrm{cm}^{-1}\right)$ for the photoelectron peaks labelled A-E in Fig. 4, given to the nearest $10 \mathrm{~cm}^{-1}$, and assignments for the ion states that make the largest contribution to each peak at $0 \mathrm{ps}$.

\begin{tabular}{|c||c|c|c|c|c|c|c|c|c|c|}
\hline \begin{tabular}{c}
\hline S 1 \\
state
\end{tabular} & \multicolumn{2}{|c|}{$\mathrm{A}$} & \multicolumn{2}{|c|}{$\mathrm{B}$} & \multicolumn{2}{c|}{$\mathrm{C}$} & \multicolumn{2}{c|}{$\mathrm{D}$} & \multicolumn{2}{c|}{$\mathrm{E}$} \\
\hline \hline $\begin{array}{c}\mathrm{pFT} \\
13^{1} 1^{1}\end{array}$ & 1230 & $13^{1}$ & 1670 & $13^{1} 6 a^{1}$ & 2060 & $13^{1} 1^{1}$ & 2490 & $13^{1} 1^{1} 6 a^{1}$ & 2870 & $13^{1} 1^{2}$ \\
\hline $\begin{array}{c}\mathrm{pFT} \\
7 a^{1} 1^{1}\end{array}$ & 1330 & $7 a^{1}$ & 1770 & $7 a^{1} 6 a^{1}$ & 2160 & $7 a^{1} 1^{1}$ & 2610 & $\begin{array}{c}7 a^{1} 1^{1} 6 a^{1} \\
+7 a^{2}\end{array}$ & 2970 & $7 a^{1} 1^{2}$ \\
\hline $\begin{array}{c}\mathrm{pDFB} \\
7 a^{1} 1^{1}\end{array}$ & 1380 & $7 a^{1}$ & 1810 & $7 a^{1} 6 a^{1}$ & 2210 & $7 a^{1} 1^{1}$ & 2670 & $7 a^{1} 1^{1} 6 a^{1}$ & 3030 & $7 a^{1} 1^{2}$ \\
\hline
\end{tabular}

Table II: IVR lifetimes $\left(\tau_{\text {IVR }}\right)$, oscillation periods $\left(\bar{\tau}_{\text {osc }}\right)$ and eigenstate energy separations $(\Delta E)$ determined for selected $\mathrm{S}_{1}$ vibrational levels in $\mathrm{pFT}$ and pDFB.

\begin{tabular}{|c|c|c|c|c|c|c|}
\hline \multirow{2}{*}{ Molecule } & \multirow{2}{*}{ Level } & \multirow{2}{*}{$\begin{array}{c}\mathrm{S}_{1} \text { energy } \\
\mathrm{cm}\end{array}$} & \multicolumn{2}{|c|}{$\tau_{\text {IVR }} / \mathrm{ps}$} & $\bar{\tau}_{\text {osc }} / \mathrm{ps}^{\mathrm{a}}$ & \multirow{2}{*}{$\Delta E / \mathrm{cm}^{-1}$} \\
\cline { 4 - 5 } $\mathrm{pFT}$ & $13^{1} 1^{1}$ & 1990 & $18.1 \pm 3.4$ & - & $4.94 \pm 0.01$ & $6.75 \pm 0.02$ \\
\hline $\mathrm{pFT}$ & $7 \mathrm{a}^{1} 1^{1}$ & 2026 & $7.0 \pm 1.5$ & $3.4 \pm 0.5$ & $5.07 \pm 0.08$ & $6.58 \pm 0.10$ \\
\hline $\mathrm{pDFB}$ & $7 \mathrm{a}^{1} 1^{1}$ & 2068 & $\infty$ & 97 & $\begin{array}{c}205,103,9.2, \\
8.8,8.5,8.1\end{array}$ & $\begin{array}{c}0.16,0.32,3.6, \\
3.8,3.9,4.1\end{array}$ \\
\hline
\end{tabular}

a Values for pFT are from this work; those for pDFB are from Ref. 5. Because a "restricted" IVR mechanism is observed in PDFB, the IVR lifetime can be considered to be infinite.

${ }^{b}$ Values from chemical timing experiments by Parmenter's group. The value for $\mathrm{pFT}$ is the reciprocal of the IVR rate given in Ref. 12 whilst the $\tau_{\text {IVR }}$ value for pDFB is taken directly from Ref. 22. 
Table III: IVR lifetimes $\left(\tau_{\text {IVR }}\right)$, oscillation periods $\left(\bar{\tau}_{\text {osc }}\right)$ and eigenstate energy separations $(\Delta E)$ determined using picosecond photoelectron imaging spectroscopy following the excitation of five bright vibrational states in $\mathrm{S}_{1} \mathrm{pFT}$; see text.

\begin{tabular}{|c|c|c|c|c|}
\hline Level & $\begin{array}{c}\mathrm{S}_{1} \text { energy } / \\
\mathrm{cm}^{-1}\end{array}$ & $\tau_{\mathrm{IVR}} / \mathrm{ps}$ & $\bar{\tau}_{\text {osc }} / \mathrm{ps}$ & $\Delta E / \mathrm{cm}^{-1}$ \\
\hline \hline $1^{1}$ & $\sim 800$ & $\infty$ & $5.0,5.4^{\mathrm{a}}$ & $6.7,6.2^{\mathrm{a}}$ \\
\hline $13^{1}$ & 1194 & $50^{\mathrm{b}}$ & - & - \\
\hline $7 \mathrm{a}^{1}$ & 1230 & 13 & - & - \\
\hline $13^{1} 1^{1}$ & 1990 & 18 & 4.9 & 6.8 \\
\hline $7 \mathrm{a}^{1} 1^{1}$ & 2026 & 7 & 5.1 & 6.6 \\
\hline
\end{tabular}

a Values taken from Ref. 3.

${ }^{\text {b }}$ Value taken from Ref. 6.

Table IV: Atomic separations corresponding to a methyl hydrogen atom $\left(\mathrm{H}_{\mathrm{m}}\right)$ and the closest ring hydrogen $\left(\mathrm{H}_{\mathrm{r}}\right)$ and carbon $\left(\mathrm{C}_{\mathrm{r}}\right)$ atom, and the net van der Waals interaction energies resulting from the sum of pairwise interactions between the methyl and nearby ring atoms in pFT. These parameters are calculated at equilibrium and at two extremes of vibrational motion, (i) and (ii), for each of modes 1,13 and 7 .

\begin{tabular}{|c|c|c|c|c|c|c|c|}
\hline \multirow[b]{3}{*}{$R\left(\mathrm{C}_{\mathrm{r}}-\mathrm{H}_{\mathrm{m}}\right)_{\min } / \AA$} & \multirow{2}{*}{ Equilibrium } & \multicolumn{2}{|c|}{ Mode 1} & \multicolumn{2}{|c|}{ Mode 13} & \multicolumn{2}{|c|}{ Mode 7a } \\
\hline & & (i) & (ii) & (i) & (ii) & (i) & (ii) \\
\hline & 2.67 & 2.78 & 2.60 & 3.07 & 2.27 & 2.74 & 2.60 \\
\hline$R\left(\mathrm{H}_{\mathrm{r}}-\mathrm{H}_{\mathrm{m}}\right)_{\min } / \AA$ & 2.46 & 2.44 & 2.51 & 2.34 & 2.59 & 2.91 & 2.02 \\
\hline$E_{\mathrm{vdw}}{ }^{\mathrm{a}} / \mathrm{kJ} \mathrm{mol}^{-1}$ & 1.63 & 0.70 & 2.57 & 0.91 & 14.20 & -0.55 & 16.95 \\
\hline$E_{v d w^{a}} / \mathrm{cm}^{-1}$ & 137 & 59 & 215 & 76 & 1187 & -46 & 1417 \\
\hline$\Delta E_{\mathrm{vdw}} \mathrm{b}^{\mathrm{b}} / \mathrm{cm}^{-1}$ & 0 & \multicolumn{2}{|c|}{156} & \multicolumn{2}{|c|}{1111} & \multicolumn{2}{|c|}{1463} \\
\hline
\end{tabular}

${ }^{\mathrm{a}} E_{\mathrm{vdw}}$ is the net van der Waals interaction energy that occurs at the listed configuration and is calculated using the van der Waals parameters given in Ref. 34 .

${ }^{\mathrm{b}} \Delta E_{\mathrm{vdw}}$ is the maximum change in van der Waals energy that occurs during the vibrational motion 


\section{Figures}

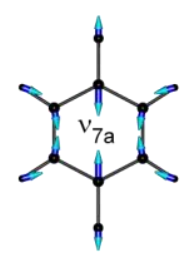

pDFB

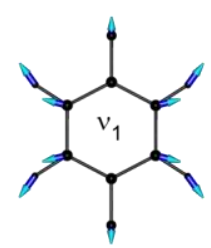<smiles>CCC1C(C)C(C)C(CC)C(C(CC)C(C)(C)C)C1CC</smiles>

$\mathrm{pFT}$<smiles>CC1=C(C)C(C(C)(C)C)C(C)(C)C1C</smiles>

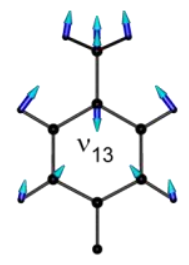

$\mathrm{pFT}$<smiles>CC=C1C(CC)C(CC)C(C(C)C)C(C(C)CC)C1CC</smiles>

Fig. 1: Schematic diagrams of calculated vibrational modes $7 a$ and 1 in $S_{1}$ pDFB and modes $7 \mathrm{a}, 1,13$ and $9 \mathrm{~b}$ in $\mathrm{S}_{1} \mathrm{pFT}$. The calculations were performed at the CIS/6-311G** level of theory using Gaussian 03 . The arrows provide vector representations of the atomic displacements and a lower threshold of $0.1 \AA$ has been used.

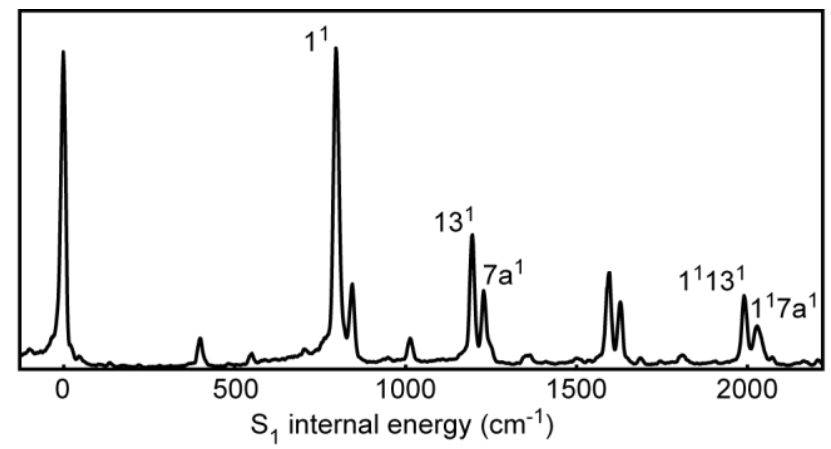

Fig. 2: $A\left(1+1^{\prime}\right)$ Resonance-enhanced multiphoton ionization (REMPI) spectrum via the $S_{1}$ excited state in pFT obtained using 1 ps laser pulses with a pump-probe time delay of $+10 \mathrm{ps}$ and a fixed probe wavelength of $284.3 \mathrm{~nm}$. Total photoelectron intensity is plotted versus $\mathrm{S}_{1}$ internal energy. Labels are included for the $13^{1} 1^{1}$ and $7 a^{1} 1^{1} S_{1}$ states that lie at $\sim 2000 \mathrm{~cm}^{-1}$ and are studied in this work, as well as those peaks assigned to fundamentals of the contributing modes 1,13 and $7 \mathrm{a}$. 
(a)

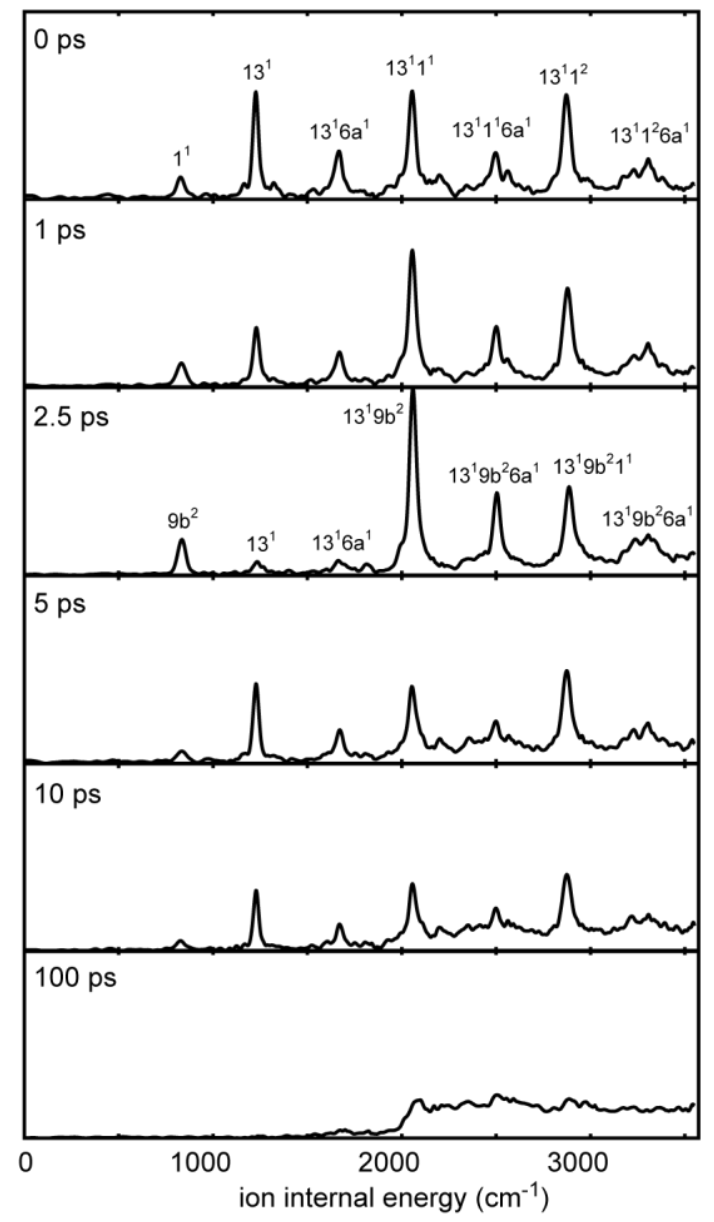

(b)

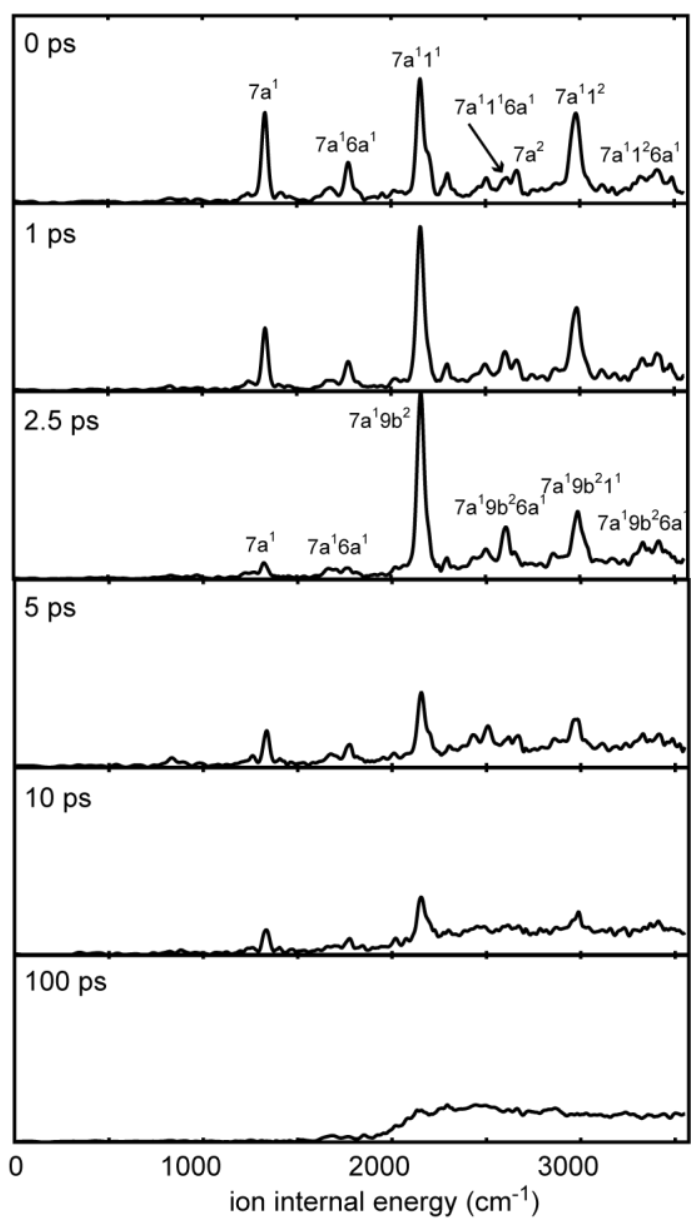

Fig. 3: Slow electron velocity-map imaging (SEVI) spectra measured via (a) $13^{1} 1^{1}$ and (b) $7 a^{1} 1^{1}$ in $\mathrm{S}_{1} \mathrm{pFT}$ at selected time delays. 
(a)

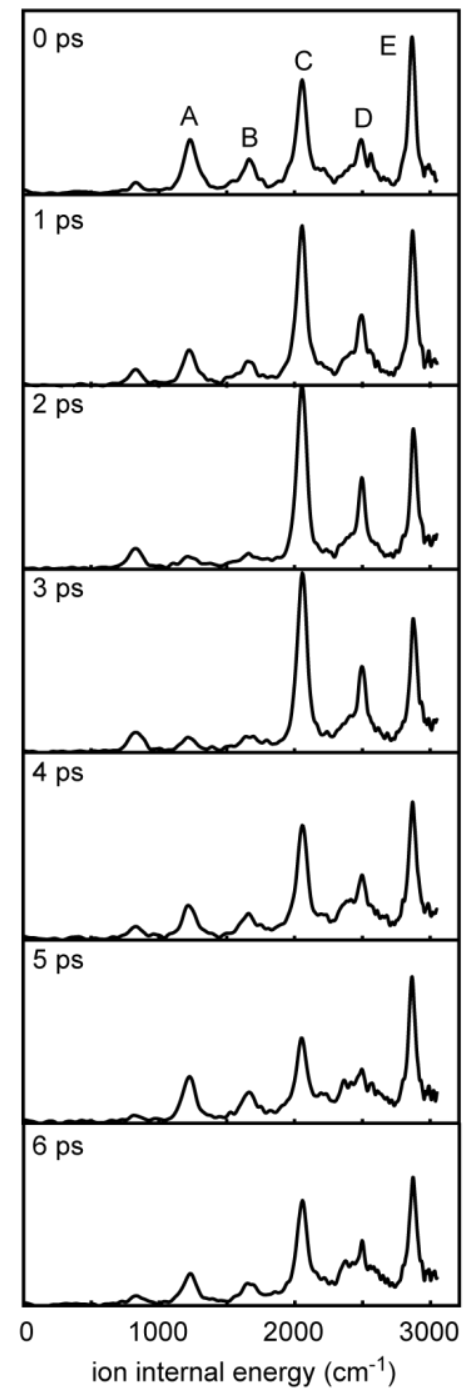

(b)

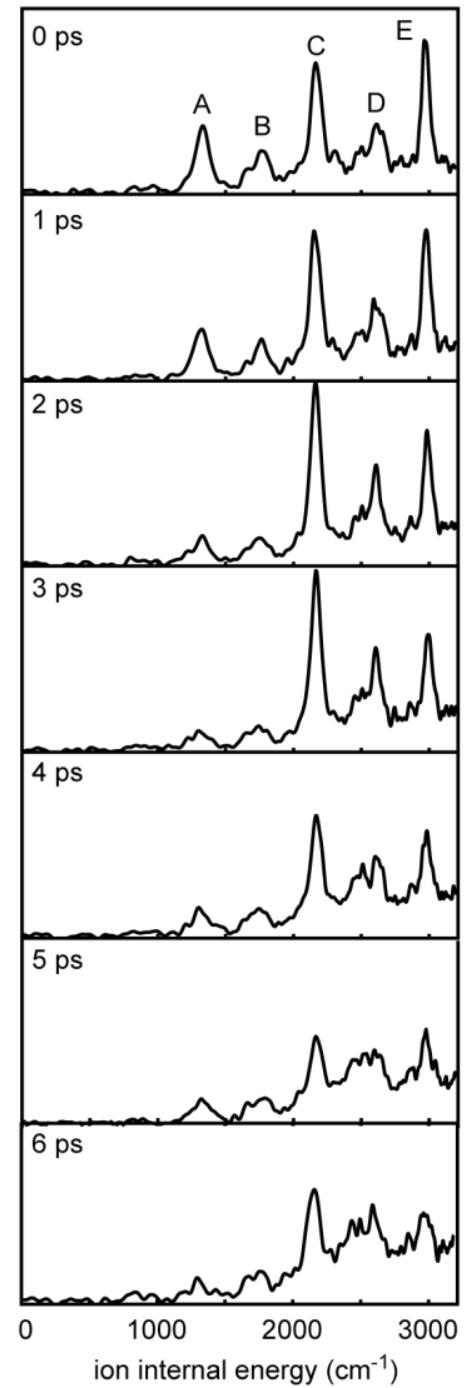

(c)

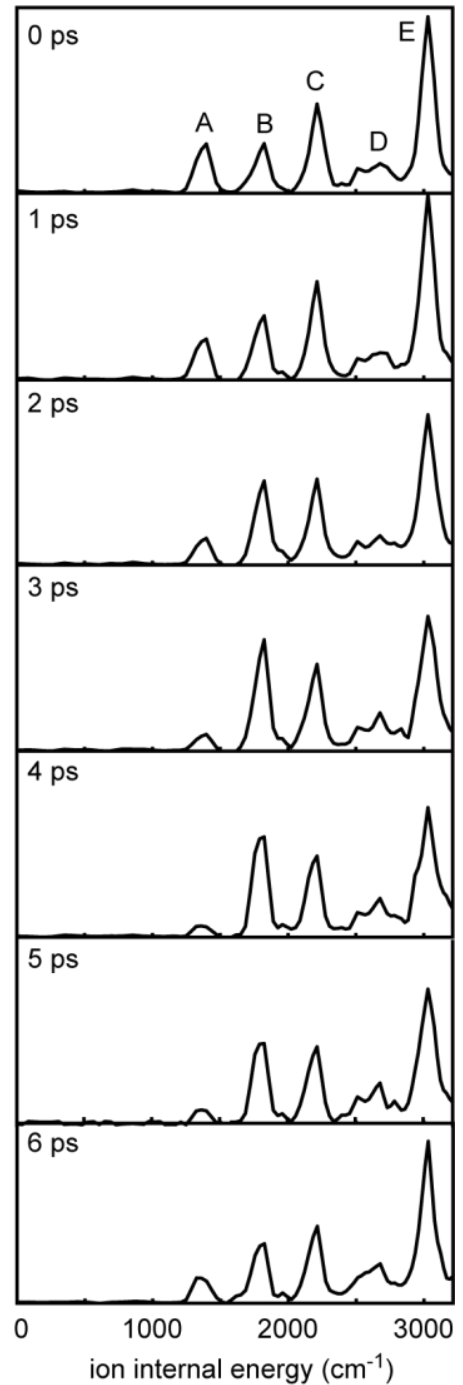

Fig. 4: Photoelectron spectra at selected early time delays following the excitation of (a) the $13^{1} 1^{1}$ vibrational state in $S_{1} p F T$, (b) the $7 a^{1} 1^{1}$ vibrational state in $S_{1} p F T$ and (c) the $7 a^{1} 1^{1}$ vibrational state in $\mathrm{S}_{1}$ pDFB. The probe wavelengths were 284.30, 283.30 and $255.75 \mathrm{~nm}$ for the spectra in panels $a, b$, and c, respectively. Assignments of the peaks labelled $A$ to $E$ are given in Table I. 
(a)

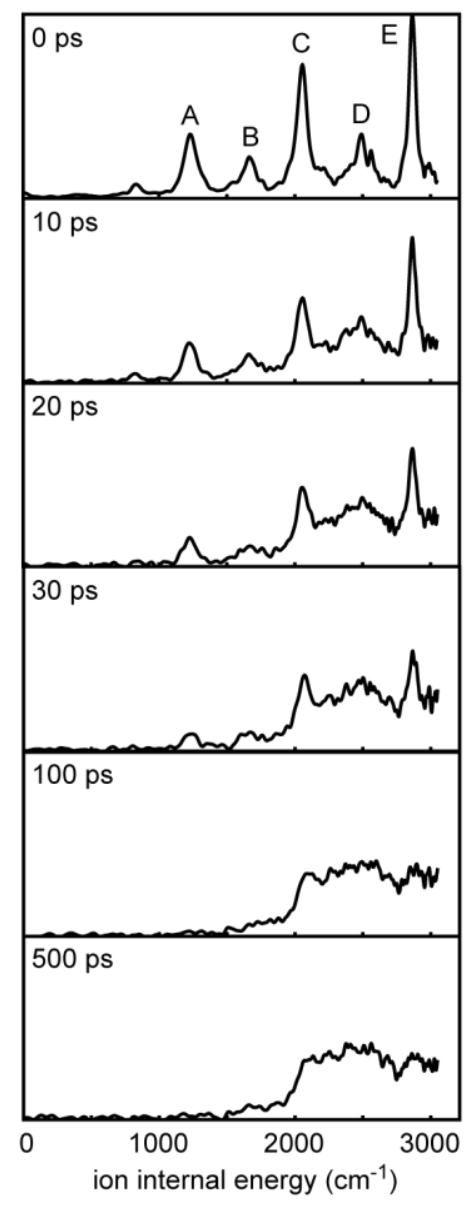

(b)

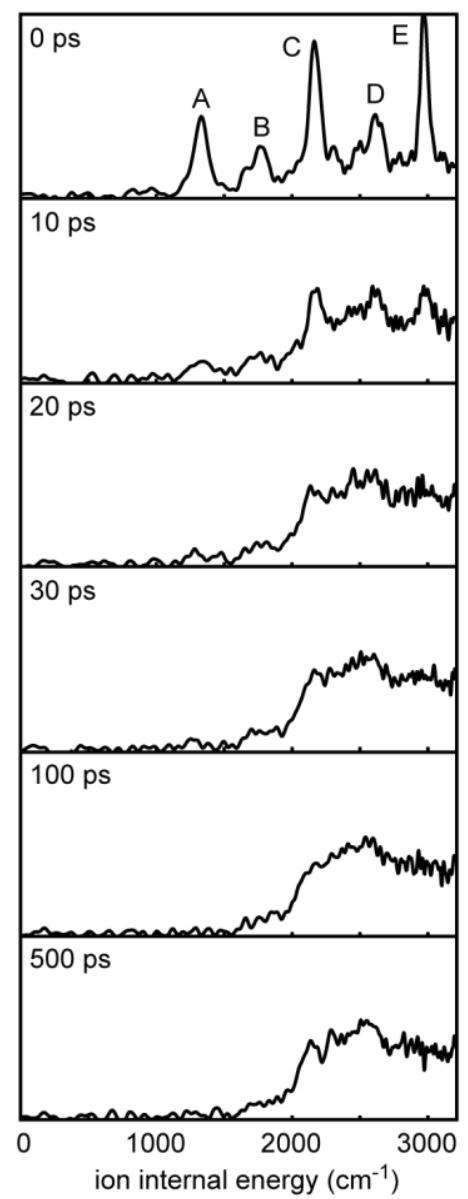

(c)

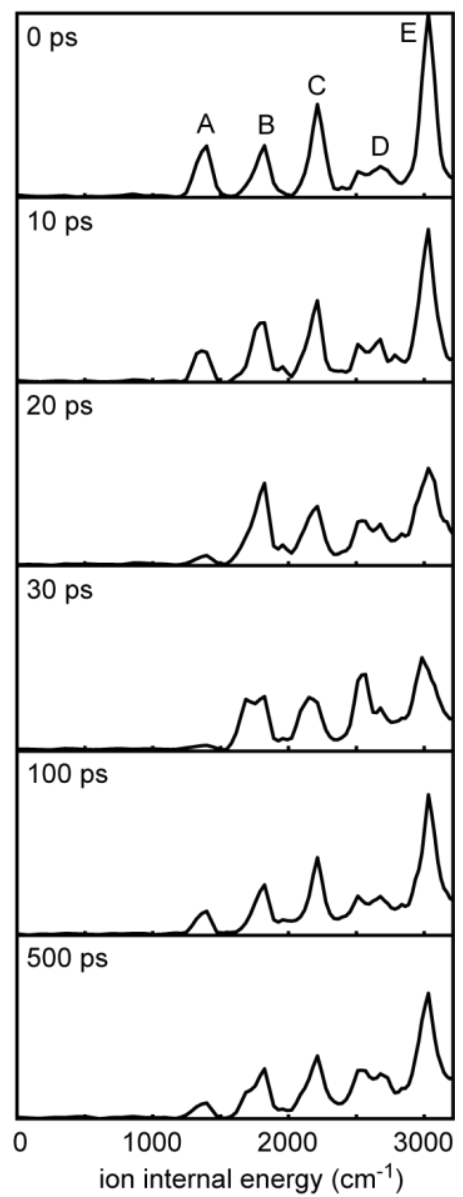

Fig. 5: As for Fig. 4 but for later time delays up to 500 ps. The photoelectron spectrum at zero time is included for comparison. 
(a)

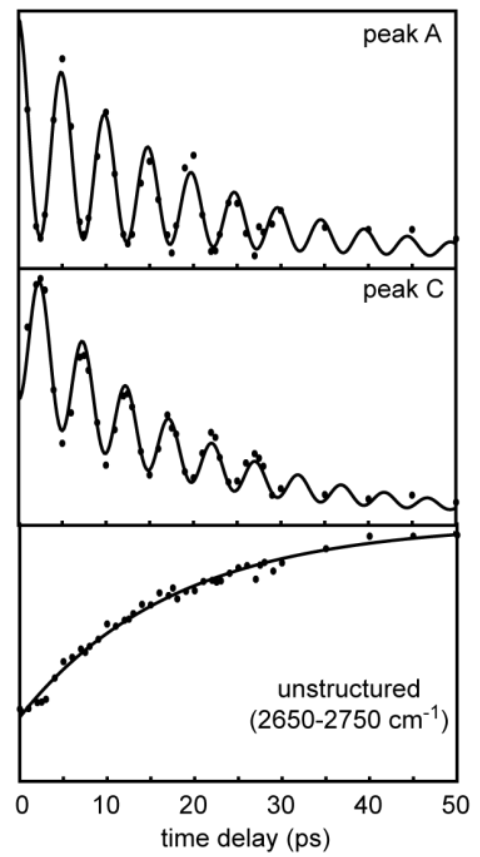

(b)

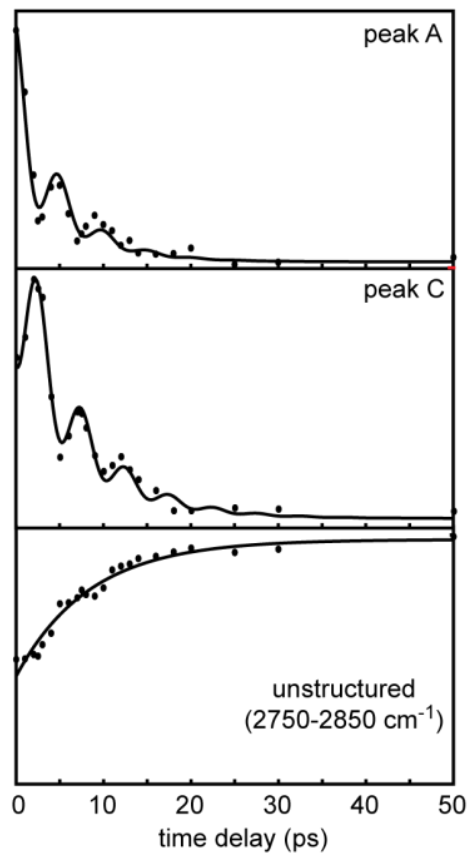

(c)

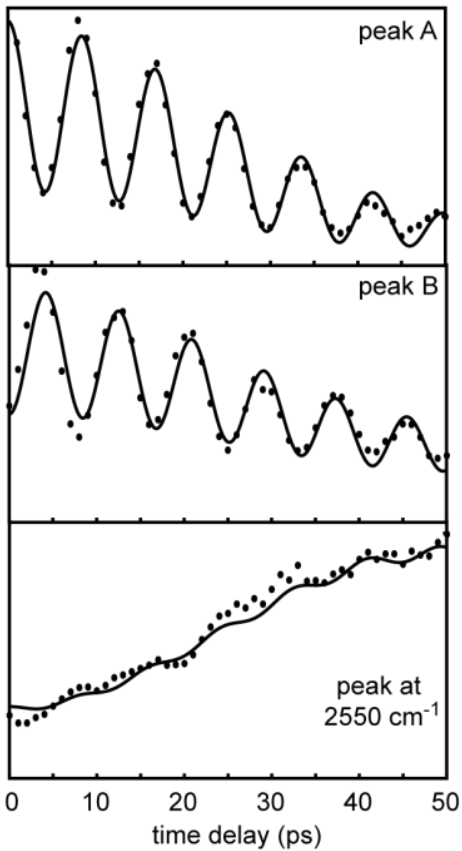

Fig. 6: Plots showing the time-dependent intensities of selected peaks and unstructured regions in the photoelectron spectra for the (a) $13^{1} 1^{1}$ vibrational state in $S_{1} \mathrm{pFT}$, (b) $7 a^{1} 1^{1}$ state in $\mathrm{S}_{1} \mathrm{pFT}$ and (c) $7 \mathrm{a}^{1} 1^{1}$ state in $\mathrm{S}_{1} \mathrm{pDFB}$. The filled circles represent experimental data points, and the solid lines represent fits. All intensity ranges start at zero but a different intensity scale is used for each panel so that changes in photoelectron peak intensities with time are easily observed. 

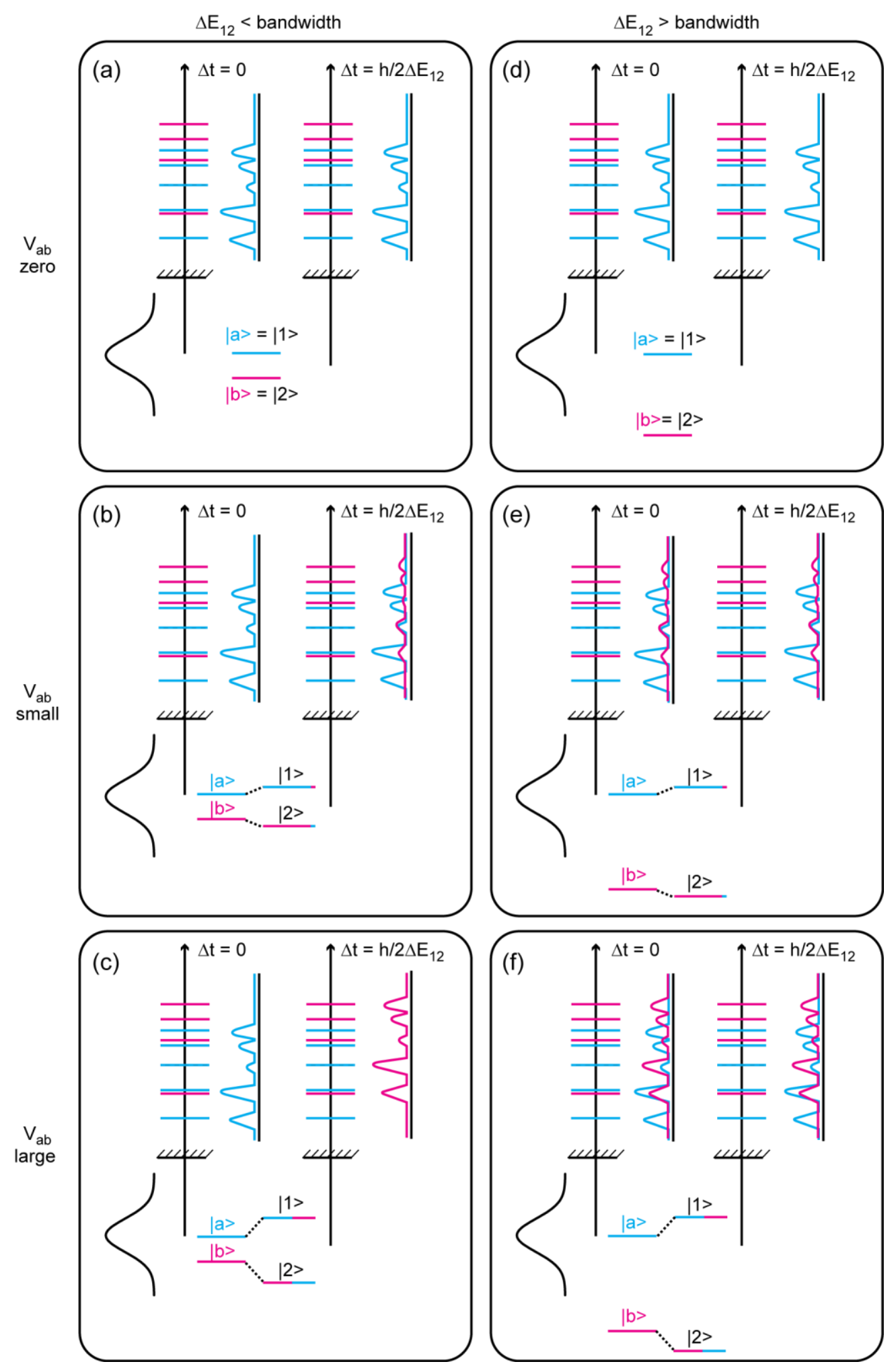

Fig. 7: A cartoon to illustrate various scenarios that can occur (see Section 4A). In all cases the bright zero order state is labelled $\mid a>$ and a dark zero order state to which it is coupled is labelled $|b\rangle$. States $\mid a>$ and $\mid b>$ each have a characteristic photoelectron spectrum (cyan and magenta). The resulting eigenstates are labelled $|1\rangle$ and $|2\rangle$. If the eigenstates both lie within the bandwidth of the pump pulse (panels (a), (b) and (c)) then they are coherently excited. In principle this can lead to time-dependence in the observed photoelectron spectra which are illustrated at $\Delta \mathrm{t}=0$ and at $\Delta \mathrm{t}=\mathrm{h} / 2 \Delta \mathrm{E}_{12}$, where $\Delta \mathrm{E}_{12}$ is the energy 
separation of $\mid 1>$ and $|2\rangle$. However, this will only be observed if there is significant coupling between $|a\rangle$ and $|b\rangle$. In panel $(a)$ the coupling is zero $\left(V_{a b}=0\right)$ and so eigenstate $|2\rangle$ has no bright state character. In this case $|b\rangle$ cannot acquire any population. In panel (c) (strong coupling) eigenstate $|2\rangle$ has $50 \%$ bright character and $|\mathrm{b}\rangle$ will acquire significant population causing the photoelectron spectra to oscillate strongly with a period inversely proportional to the eigenstate energy separation. In panels (d), (e) and (f) the eigenstate separation is larger than the bandwidth of the pump pulse. In these cases there is no coherent excitation and so no time dependence can result, but if there is significant coupling the photoelectron spectra will show both bright and dark state character at all time delays.

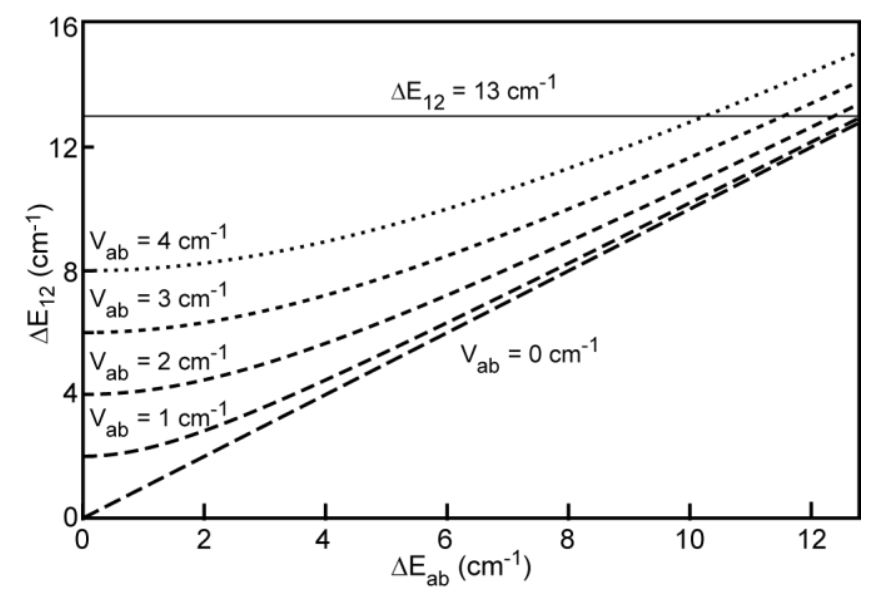

Fig. 8: The dependence of the eigenstate energy separation, $\Delta E_{12}$, on the zero-order state separation, $\Delta E_{a b}$, for selected values of the Hamiltonian coupling matrix element, $V_{a b}$. For a strong coupling limit of $V_{a b}=3 \mathrm{~cm}^{-1}$, a value of $\Delta E_{a b}>11 \mathrm{~cm}^{-1}$ is required to obtain $\Delta E_{12}>13$ $\mathrm{cm}^{-1}$. 


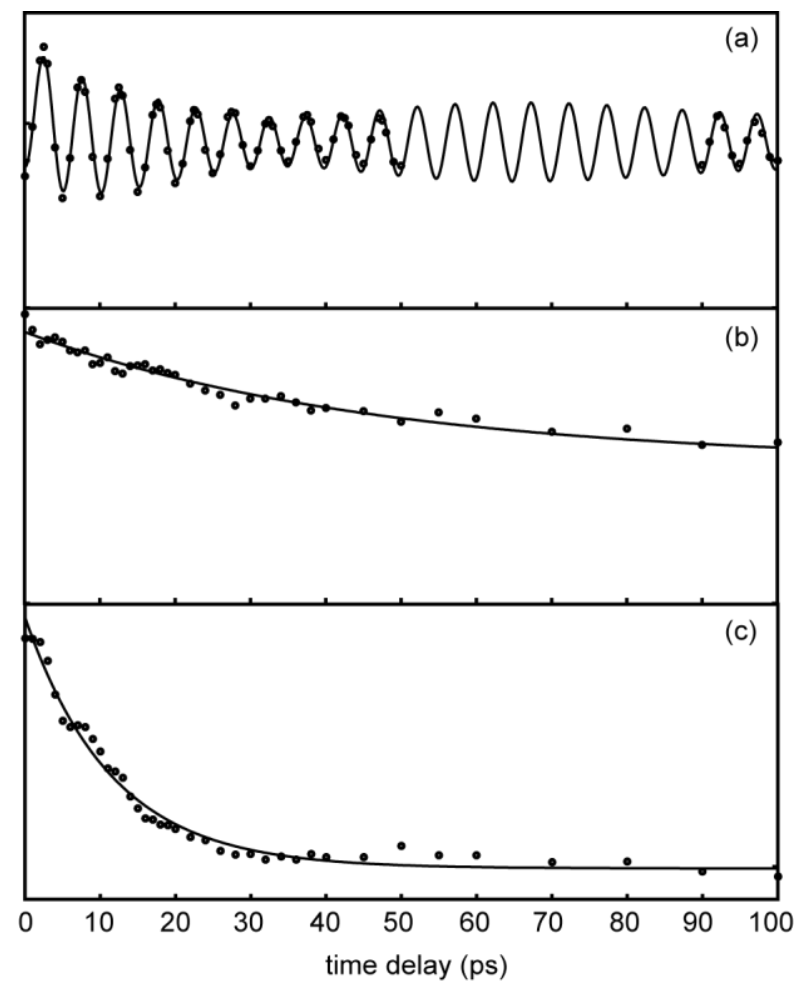

Fig. 9: Plots showing the time-dependent intensities for the $\Delta v=0$ transitions in photoelectron spectra measured via the (a) $1^{1}$, (b) $13^{1}$ and (c) $7 a^{1}$ vibrational states in $\mathrm{S}_{1}$ pFT. All intensity ranges start at zero so that the relative change in peak height is seen with respect to time. 